\title{
Übersetzungsprobleme literarischer Werke bei bilingualen Studierenden: Eine Fehleranalyse anhand Stefan Zweigs Romanen
}

\author{
Dr. Derya Perk \\ Ankara Üniversitesi, DTCF \\ Alman Dili ve Edebiyatı Anabilim Dalı \\ der@ankara.edu.tr
}

\author{
Prof. Dr. Dursun Zengin \\ Ankara Üniversitesi, DTCF \\ Alman Dili ve Edebiyatı Anabilim Dalı \\ zengin@ankara.edu.tr
}

\begin{abstract}
Bilinguale sind fähig, Inhalte in zwei Sprachen zu verstehen und in beiden Sprachen zu kommunizieren. Bei einem Übersetzungsprozess reicht es jedoch nicht, zwei Sprachen zu beherrschen, da die Übertragung eines Ausgangstextes in die Zielsprache außer Sprachwissen weitere Kompetenzen erfordert. Insbesondere in Übersetzungen von literarischen Texten, in denen der Stil und die Gattung sehr bedeutend sein können, bei Bilingualen häufig Fehler auftreten. Diese Arbeit hat es zum Ziel, eine Fehleranalyse anhand literarischen Übersetzungen $\mathrm{zu}$ machen und die von Bilingualen häufig geführten Fehlerarten festzustellen. Dafür werden aus drei verschiedenen Universitäten Studierende ausgewählt und von ihnen erwartet, dass sie Textteile aus Stefan Zweigs verschiedenen Werken übersetzen. Die Studierenden übersetzten insgesamt zehn Sätze, die aus stilistischer, orthographischer, semantischer, syntaktischer, morphologischer und pragmatischer Perspektive bewertet werden. Apropos diesem Verfahren werden auch auf die Entsprechungstypen Werner Kollers geachtet.
\end{abstract}

Keywords: Literarische Übersetzung, Bilingualität, Stefan Zweig, Fehleranalyse.

\section{Translation problems of literary works for bilingual students: A mistake analysis using Stefan Zweig's novels}

\section{Öz}

Bilinguals are able to understand content in two languages and to communicate in both languages. In a translation process, however, it is not enough to be able to speak two languages, because the translation of an original text into the target language requires additional skills as well as language knowledge. Especially in translations of literary texts, in which the style and the genre are very important, errors can often occur in bilinguals. The aim of this work is to carry out an error analysis on the basis of literary translations and to determine the types of errors frequently made by bilinguals. Students are selected from three different universities and are expected to translate parts of the texts from Stefan Zweig's different works. The students translated ten sentences in total, which are assessed from a stylistic, orthographic, semantic, syntactic, morphological and pragmatic perspective. Speaking of this procedure, attention is also paid to the equivalence types of Werner Koller. 
Anahtar Kelimeler: Literary translation, bilingualism, Stefan Zweig, error analysis.

\section{Edebi eserlerin çevirilerinde iki dilli öğrencilerin karşılaştıkları sorunlar: Stefan Zweig'in romanlarını üzerinden bir hata analizi}

Öz

İki dilliler, iki farklı dilde içerikleri anlayabilir ve her iki dilde de iletişim kurabilirler. Ancak bir çeviri sürecinde iki dile hakim olmak yeterli değildir, çünkü orijinal bir metnin hedef dile çevrilmesi dil bilgisi kadar ek beceriler de gerektirir. Özellikle üslup ve türün çok önemli olduğu edebi metin çevirilerinde iki dillilerde sıklıkla hatalar meydana gelebilmektedir. Bu çalışmanın amacı, edebi çeviriler bazında bir hata analizi yapmak ve iki dilli kişilerin sıklıkla yaptığı yanlış türlerini belirlemektir. Bunun için üç farklı üniversiteden iki dilli öğrenciler seçilmiş ve Stefan Zweig'in farklı eserlerinden çeviriler yapmaları istenmiştir. Bu bağlamda öğrenciler toplam on cümleyi çevirmiş ve bu cümleler stilistik, ortografik, anlambilimsel, sözdizimsel, morfolojik ve pragmatik bir bakış açısıyla değerlendirilmiştir. Bunun yanı sıra çevrilmiş cümleler Werner Koller'in eşdeğerlilik türleri üzerinden de yorumlanmıştır.

Anahtar Kelimeler: Edebi çeviri, iki dillilik, Stefan Zweig, hata analizi. 


\section{Feststellung des Untersuchungsgegenstands und der Untersuchungsgruppe}

Einen literarischen Text in der Zielsprache mit all seinen Eigenschaften wiederzugeben braucht bestimmte Kenntnisse, zu denen Sprachwissen, Normwissen und Kompetenz gehören. In den Abteilungen Germanistik und Übersetzungsausbildung der verschiedenen Universitäten in der Türkei werden in Übersetzungsseminaren verschiedene literarische Textteile vom Deutschen ins Türkische und vom Türkischen ins Deutsche übersetzt. In diesen Seminaren lesen die Studierenden den Text durch, forschen über die Gattungseigenschaften wie auch über den Autor und übersetzen den Text später in die Zielsprache. Die Übersetzungen werden zuletzt meistens nach ihrer semantischen und syntaktischeren Äquivalenz bewertet. In diesem Rahmen ist zu unterstreichen, dass sich diverse Übersetzungsniveaus zeigen können, da die Studierenden über verschiedene sprachliche Grundlagen verfügen. In den erwähnten Abteilungen ist eine Gruppe von Studierenden, die die deutsche Sprache in der türkischsprachigen Umgebung, zum Teil im Gymnasium (meisten bis zum A2 Niveau) und später in der Vorbereitungsklasse (bis zum B2 Niveau) der Universitäten gelernt haben. Die zweite Gruppe, besteht aus Bilingualen, die in Deutschland geboren sind und/oder die deutsche Sprache mit Umgang mit Muttersprachlern in kleinem Alter erworben haben. Bei der literarischen Übersetzung zeigen sich bei beiden Gruppen Fehler, die aus verschiedenen Gründen entstehen können. Zu unterstreichen ist, dass auch die zweite Gruppe nicht immer einen Übersetzungsprozess eines literarischen Textes fehlerlos abschließen kann. Sie beherrschen zwar die grammatischen Normen beider Sprachen, können aber das Gesagte und/oder Geschriebene nicht immer äquivalent übersetzten. Ein Grund für diese Problematik könnte das "linguistische Relativitätsprinzip" sein, das wie folgt erklärt wird:

"Aus der Tatsache der Strukturverschiedenheit der Sprachen folgt, was ich das

"linguistische Relativitätsprinzip" genannt habe. Es besagt, grob gesprochen, folgendes: Menschen, die Sprachen mit sehr verschiedenen Grammatiken benutzen, werden durch diese Grammatiken zu typisch verschiedenen Beobachtungen und verschiedenen Bewertungen äußerlich ähnlicher Beobachtungen geführt. Sie sind daher als Beobachter einander nicht äquivalent, sondern gelangen zu irgendwie verschiedenen Ansichten von der Welt." (E. Sapiers nach Koller, 2001, s. 171)

Diese Studie geht deshalb der Frage nach, welche Fehler beim Übersetzungsprozess ohne Hilfsmaterial bei bilingualen Studierenden erscheinen und aus welchen Gründen sie entstanden sind.

Bilinguale Studierende, die an der Ankara Universität (Ankara), Hacettepe Universität (Ankara), Akdeniz Universität (Antalya) Germanistik studieren übersetzen in diesem Zusammenhang Textteile aus Stefan Zweigs Werken. Die von ihnen übersetzten Textteile aus Stefan Zweigs Werken - Angst, Die Mondscheingasse (DM), Die Wunder des Lebens (DWDL), Untergang eines Herzens (UEH) - werden aus syntaktischer, semantischer, morphologischer, pragmatischer, stilistischer und orthographischer Perspektive und nach Kollers (2001) Entsprechungstypen betrachtet.

\section{Ein genereller Überblick zur literarischen Übersetzung}

Die Literatursprache ist im Gegenteil zu den anderen schriftlichen Textsorten die Sprache, die auf einem Dichter beruht und die als ein Zeichen der "hochentwickelten 
Kulturform" erklärt wird (Best, 2004, s. 313). Bei einem Übersetzungsprozess sind somit die Ausdrucksweise des Dichters bzw. sein Stil und die erwünschte Funktion des Textes von großer Bedeutung. Wichtig ist in diesem Bezug, ob der Autor oder der Erzähler sich an den Leser wendet, also wer sich als Sprecher artikuliert. Greiner (2004, s. 14ff) nähert sich an das Thema, in dem er sich an Eugene E. Nidas Definitionen anlehnt. Befragt wird in diesem Zusammenhang zuerst, ob es möglich ist, eine Übersetzung als richtig oder falsch zu bewerten und sich an Kriterien wie Nidas zu halten. Die nächste Fragestellung ist die Erklärung der Übersetzungen von literarischen Texten als ein kommunikativer Akt. In diesem Fall erreicht die Romanlektüre als Sender (S) einen Empfänger (E), aber die Frage, ob der Autor oder der Erzähler des Textes sich fiktiv an den Leser wendet, bringe dieses Argument zu einem Dilemma. Der Text sollte nicht als referentielle-Sprache akzeptiert und die Wahrheit einer Aussage nicht befragt werden, denn in "Sprachkunstwerken bildet Sprache eine Sinnstruktur, ein literarischer Text ist eine sich selbst genügende Sinnstruktur". Eine nächste Feststellung ist, dass der Materialcharakter der Sprache beim Übersetzen sehr wichtig ist und die Bedeutung keine Priorität gegenüber dem Stil haben kann. Auch wird der Gedanke, dass das Übersetzte nicht fremd wirken soll, in Frage gestellt. Die Literatur als fiktives Produkt sei dem Leser fremd durch seine neue und ungewöhnliche Wirkung, sonst wäre sie nur trivial (Nida nach Greiner, 2004, s. 14ff). Reiß erklärt einen literarischen Text als einen expressiven Texttyp, dessen Textfunktion die künstlerische Aussage ist und die sich auch durch eine senderorientierte Stellung kennzeichnet. Während das Ziel der Übersetzung demnach die Analogie der künstlerischen Gestaltung ist, ist die Methode der Übersetzung "identifizierend" (nach Stolze, 2008, s. 114). Dies bedeutet natürlich nicht, dass es sich um eine freie Übersetzung handelt. Wobei die freie Übersetzung zu einem Sprachkunstwerk führt, die beim Leser dieselbe Wirkung des AT bzw. dem Original erzielt (Reinart, 2009, s. 164). Ob es hier um die illusionistischen Methoden J.Levýs' handelt, die beim Leser das Gefühl erwecken, als lese er einen Originaltext, ist eine offene Frage, denn nach Levý ist unter diesem Terminus nicht eine Adaptation zu verstehen, die die eigentliche Fremdheit beseitigt (nach Koller, 2001, s. 295). Er beschreibt die illusionistische Übersetzung aus seinen Vorstellungen wie folgt:

\begin{abstract}
"Der illusionistische Übersetzer verbirgt sich hinter dem Original, das er gleichsam ohne Mittler dem Leser mit dem Ziel vorlegt, bei ihm eine übersetzerische Illusion zu wecken, die Illusion nämlich, daß er die Vorlage lese [...] der Romanleser weiß, daß er eine gedachte Geschichte lies, aber er fordert, daß der Roman sich an die Regeln der Wahrscheinlichkeit hält. So weiß auch der Leser einer Übersetzung, daß er nicht das Original liest, aber er verlangt, daß die Übersetzung die Qualität des Originals beibehalte." (Levy nach Stolze, 2008, s. 138).
\end{abstract}

Bei einer literarischen Übersetzung sollten die gesamten Eigenschaften als Referenz des Prozesses betrachtet werden, da wie erwähnt auch der Stil des Autors ein wichtiges Faktum ist. Denn „der Materialcharakter der Wörter, Wort- und Satzverknüpfungen gewinnen ebenso sinnkonstituierende Dimensionen wie auch der Textsinn sich nur in der spezifischen Form des Textes konstituiert" (Greiner, 2004, s. 19-20).

Abgesehen von dem neuen Blick auf die pragmatische Einbettung des gesamten Übersetzungsvorgangs, der nach Stolze (2008, s. 138) ein wichtiger Bestandteil ist, sind die verschiedenen Äquivalenzarten bei dieser Arbeit, deren Gegenstand ein literarischer Text und die ÜbersetzerInnen bilinguale Germanistikstudenten sind, ein bedeutender Bestandteil. Um eine gelungene und treue Übersetzung $\mathrm{zu}$ erreichen sollte bei dieser 
Zielgruppe eine Äquivalenzrelation geführt werden, die nach Koller auf die Übersetzungsbeziehung zwischen zwei Texten weist. Koller beschreibt in diesem Zusammenhang fünf Entsprechungstypen im lexikalischen Bereich: Eins-zu-eins, Eins-zuviele, Viele-zu-eins, Eins-zu-Null, Eins-zu-Teil (Koller,2001,s.228). Auch diese Entsprechungstypen sind im Übersetzungsprozess entscheidend, neben der "neu aufgebauten" Wiedergabe in der ZS ist auch die Äquivalenz des AT und ZT zu beachten, denn im Gesamten transportiert eine Übersetzung „die sprachlichen Inhalte einer Muttersprache in solche einer anderen Muttersprache, die beide je unterschiedliche geistige Zwischenwelten konstituieren, in denen die "Welt" dem Menschen verfügbar und kommunizierbar gemacht wird“ (Koller, 2001, s. 170).

$\mathrm{Zu}$ betonen sind in diesem Zusammenhang die Fähigkeit, Fertigkeit, Kompetenz der Studierenden. Die Fähigkeit, die angeborene Anlage etwas zu erlernen und auszuführen (Stolze, 2013, s. 21), ist bei dieser Arbeit bzw. für die Studierenden nicht das bevorzugte Gut. Eher erfüllt die Fertigkeit, also das implizite, subjektive Wissen (Stolze, 2013, s. 21) der Studierenden die Erwartung, da sie als bilinguale Germanistikstudenten die sprachlichen Normen beider Sprachen gelernt und bisher mindestens literarische Textteile übersetzt haben. Die Kompetenz, die eine Kombination von Fähigkeit, Fertigkeit und Wissen ist, ist an dieser Stelle die brisanteste Eigenschaft. Natürlich sind in dieser Beziehung zwischen übersetzerischer Kompetenz (implizit übersetzerischer Kompetenz und explizitem Wissen); sozialer, kultureller und interkultureller Kompetenz (implizit aufgebautem Wissen und explizitem Wissen) und kommunikativer Kompetenz (pragmatischer und linguistischer Kompetenz) zu unterscheiden (Hansen, 1999, s. 341). Auch wenn die teilnehmenden bilingualen Studierenden die Fähigkeit -wenn auch zum Teil- besitzen, ist das nicht immer ausreichend, denn neben der intensiven Beschäftigung mit der Muttersprache fördert die Übersetzung einen von kulturellem und individuellem Wissen unterstützten aktiven Sinnstiftungsprozess. Die muttersprachliche Kompetenz ist sozusagen „der zentrale Angelpunkt translatorischer Kompetenz" (Resch, 1999, s. 345).

Zuletzt sind die Übersetzungsprobleme der bilingualen Studierenden bzw. die im Übersetzungsprozess auftretenden Sprachfehler zu nennen. Nach der Lasswell-Formel werden in dieser Kategorie ausgangsspezifische, pragmatische, kulturpaarspezifische und sprachenpaarspezifische Übersetzungsprobleme genannt (nach Stolze, 2008, s. 191). In dieser Arbeit stehen bei der Interpretation von Fehlern deshalb neben den stilistischen Eigenschaften auch die syntaktische, semantische, morphologische, orthographische und textnormative Äquivalenzerscheinung im Fokus. Außerdem werden bei der Bewertung die Wiedergabe der pragmatischen und kommunikativen Eigenschaften analysiert. Die bilingualen Übersetzer werden vor dem ÜP deshalb darüber informiert, dass sie zuerst den Text erfassen, ihn interpretieren und zuletzt umformulieren sollen, wie auch nach Levý in "Der Vorgang des Übersetzens" ausgedrückt wird (vgl. Koller, 2001, s. 296).

\section{Zur Bilingualität der Studierenden}

Eine Definition zum Begriff "Bilingualität" ist nur möglich, wenn verschiedene Literaturen zum Thema auseinandergelegt werden. Wie auch Banaz (2002, s. 7) ausdrückt, wird dieser Forschungsgegenstand nämlich in einer individuellen und in einer gesellschaftlichen Ebene untersucht. Nach Lewandowski (1979, s. 123) ist unter einer eigentlichen Bilingualität jedoch zu verstehen, dass es sich um eine Fähigkeit handelt, zwei Sprachen zu beherrschen und sich in diesen Sprachen gleich gut auszudrücken bzw. zu 
verständigen. Der Erwerbsprozess von zwei Sprachen beruht auf Sprachsystemen, die sich natürlich unterscheiden, aber in vielen Fällen wie die Hauptkategorien Zeit, Modalität, Person etc. ähneln können. Die meisten Wörter haben üblich mindestens eine Entsprechung in der zweiten Sprache und vergleichbare syntaktische Regeln, die - auch wenn anders aufgebaut - zu einlernen sind. Ortega (2009, s. 4) erklärt den Erwerbsprozess der zwei Sprachen mit anderen Worten und befragt zwei Hauptpunkte mit folgenden Sätzen:

\begin{abstract}
"We use the term 'bilingual acquisition' or 'multilingual acquisition' to refer to the process of learning to or more languages relatively simultaneously during early childhood - that is, before the age of four. [...] Two key questions of interest are how the two (or more) languages are represented in the brain and how bilingual speakers switch and alternate between their two (or more languages, depending on a range of communicative needs and desires."
\end{abstract}

Klein (1992, s. 23) gibt für diese Erklärung ein Beispiel aus dem lexikalischem Bereich. Ihm nach lernt zum Beispiel ein Deutsch und Französischsprachiger im üblichen Prozess, dass es für den "Begriff 'Stuhl' zwei phonologische Relationsformen gibt, etwa [Jtu:l] und [

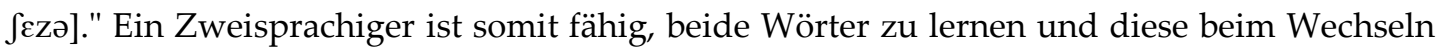
von einer Sprache zum anderen auszuwählen. Im zweiten Fall, in der ein Zweisprachiger eine Sprache als Muttersprache und die zweite Sprache als Zweitsprache gelernt hat, ändert sich die Situation, da nach einem System ein zweites aufgebaut wird und die Person nicht innerhalb eines Systems umschaltet, sondern von einem System zum zweiten (Klein, 1992, s. 23). Die Studierenden unserer Forschung sind demnach richtige Bilinguale, die beide Sprachen meistens gleichzeitig zu Hause und/oder in der Schule gelernt haben. Bei ihnen handelt es sich keineswegs um eine partielle Bilingualität, sondern eher um eine eigentliche Bilingualität (siehe zur Erklärung Löffler, 2010, s. 71-72).

Beim dieser Umwechselung von einer Sprache auf die andere Sprache handelt es sich eigentlich um eine Sprachalternation, die eine Erscheinungsform davon Mehrsprachigkeit ist. Zweisprachige bzw. Mehrsprachige "nutzen die kommunikativen Möglichkeiten, welche ihnen die Kenntnis von mehr als einer Sprache bietet" (Hacisalihoğlu, 2009, s. 91). Dies bedeutet, dass in einem Sprachkontakt auch während des Gespräches Satzteile oder Wörter der anderen Sprache eingebaut werden können. Wichtig ist hier zu wissen, dass die erst erworbene Sprache sprachstrukturell dominanter als die zweite Sprache ist. Die zweite Sprache taucht meistens im lexikalischem Bereich hervor (Riehl nach Benteler, 2019, s. 166), d.h. wenn eine Vokabel der Person nicht einfällt oder er im kulturellen Aspekt das richtige Wort nicht aussuchen kann.

\title{
3. Zu den Beispielen
}

\subsection{Beispiel 1}

">Zum Südbahnhof<, stieß sie dann hastig heraus und, plötzlich vom Gedanken erfaßt, die Person könnte ihr folgen, >rasch, rasch, fahren Sie schnell!<" (Zweig, Angst, s. 6) Übersetzungsbeispiele der Studierenden:

1. >Güney'deki tren istasyonuna<, diye çıktı ağzından, aniden kafası bastı, o kişi kendisini takip edebilirdi, >hadi, hadi, hadi basın gaza!<

2. Güney Trenistasyonuna, apar topar dışarı çıktı ve aniden düşüncesi beni yakaladı, o kişi onu takip edebilirdi. Çabuk, çabuk, hızlı sürün!

3. "Südbahnhof'a", heyecanlı bir şekilde seslendi ve aniden bir düşünce sardı, o takip ediyor 
olabilir,"hızlı, hızlı, hızlı sürün!"

4. >metro'ya< hızlı bir şekilde indi we birden aklına geldi, o kişi onu takip edebilirdi.> Cabuk, cabuk, hizlı sürün.

\section{Bewertung der Übersetzungen:}

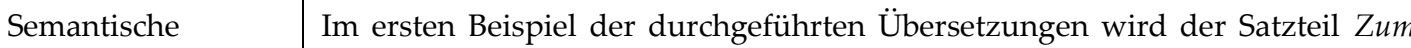

Bewertung Südbahnhof ins Türkischen semantisch nicht richtig übersetzt. Mit einer Rückübersetzung ins Deutsche ist das leicht festzustellen. Die türkische Übersetzung bedeutet so viel wie "Zum Bahnhof, der im Süden ist". Im dritten Beispiel wird das deutsche Wort Südbahnhof nicht übersetzt, sondern nur im Türkischen (Südbahnhof'a) dekliniert. Hier geht es zugleich um ein CodeSwitching, da von den deutschen Wörtern Gebrauch gemacht wird und das Nomen sogar Türkisch dekliniert wird, was auch zum morphologischen Bereich gehört. All diese Merkmale weisen selbstverständlich auf die Bilingualität hin. Das Wort Bahnhof wird als metro (D. U-Bahn) falsch übersetzt, d.h. denotativ ist keine Äquivalenz vorhanden. Die Sätze und Satzteile werden auch falsch übersetzt. Der Satzteil plötzlich vom Gedanken erfasst wird z.B. ins Türkische als aniden kafası bastı, düşüncesi beni yakaladı oder aklına geldi übertragen und die semantische Äquivalenz nicht erreicht, da die Redewendung kafası basmak beim Türkischen etwas kappieren bedeutet. Die türkische Entsprechung dieser Aussage ist nicht düşüncesi beni yakaladı, sondern düşüncesi beni sardl, d.h. soviel wie der Gedanke hat mich erfasst. Dieser Fall weist also auf die fehlenden türkischen Sprachkenntnisse. Ein weiteres Beispiel ist aklına geldi, welches hat sich daran erinnert oder es fiel ihm ein bedeutet. Der Satz wird aus diesen Gründen als semantisches Fehler bezeichnet. Daran kann man ohne weiteres erkennen, dass der bilingualen Übersetzer zugleich die deutsche Sprache nicht so gut beherrschen, da sie den Satz nicht verstehen und ihn falsch übersetzen. Denselben Fall treffen wir auch im zweiten Beispiel, in dem der Satz stieß sie dann hastig heraus als apar topar dışarı çıktı. d.h. ging eilig raus übersetzt wird. Dieses Beispiel zeigt wiederum, dass man den Satz in der Ausgangssprache nicht verstanden hat und dadurch ihn in die Zielsprache nicht äquivalent übertragen konnte. Letztendlich ist aus diesen Übersetzungen zu erkennen, dass die denotative oder konnotative Bedeutung von Wörtern oder Sätzen nicht beachtet oder nicht richtig übersetzt wird.

Orthographische Bewertung
Im zweiten Beispiel erscheint ein orthographischer Interferenzfehler Trenistasyonuna als Zeichen eines negativen Transfers. Denn das Wort wird, obwohl es klein und getrennt geschrieben werden soll, wie im Deutschen (Bahnhof) groß und zusammengeschrieben, d.h. deutsche orthographische Regeln werden ins Türkische übertragen. Der Grund dafür ist selbstverständlich vor allem die Bilingualität bzw. der Versuch, die orthographischen Regeln in die andere zu übertragen. In der letzten Übersetzung erscheinen ähnliche Beispiele zu den orthographischen Fehlern. Die türkischen Wörter ve (D. und) und çabuk (D. schnell) werden mit Buchstaben $w$ und $c$ aus dem deutschen Alphabet geschrieben. Der deutsche Laut $w$ entspricht im türkischen dem Buchstaben $v$. Außerdem besitzt das türkische Alphabet die Buchstaben $c$ und $c$, die verschiedenen Lauten 


\begin{tabular}{|l|l|}
\hline $\begin{array}{l}\text { entsprechen. Da das Laut } c ̧ \text { [t]] im deutschen nicht existiert, wird es mit dem } \\
\text { Buchstaben } c \text { transkribiert. Auf diese Weise werden die Regeln der deutschen } \\
\text { Sprache ins Türkische übertragen, wobei sie orthographische Interferenzfehler } \\
\text { verursachen, was ja vor allem auf die Bilingualität hinweist. } \\
\text { Im Bereich der Interpunktion kommen auch Fehler vor: Der deutsche Satz } \\
\text { rasch, rasch, fahren Sie schnell! ist ein Imperativsatz mit Ausrufezeichen. Der soll } \\
\text { im Grunde bei der Übersetzung mit einem Ausrufezeichen versehen werden, } \\
\text { was jedoch nicht der Fall ist. Wie es im 4. Beispiel Güney'deki tren istasyonuna } \\
\text { zu bemerken ist, hat der Satz kein Ausrufezeichen. Aus diesem Grund ist } \\
\text { dieser Satz hinsichtlich der Interpunktion nicht äquivalent. Orthographische } \\
\text { Fehlübersetzungen gehen also auf der einen Seite auf die Bilingualität der } \\
\text { Übersetzer und auf der anderen Seite auf fehlende Sprachkenntnisse im } \\
\text { orthographischen Bereich zurück. }\end{array}$ \\
\hline
\end{tabular}

\subsection{Beispiel 2}

">Donnerwetter,< sagte der Wirt ganz verwundert, >Ihr habt da ein Meisterstück gemacht.<" (Zweig, DWDL, s. 30)

Übersetzungsbeispiele der Studierenden:

Donnerwetter: Hadi be!, Hadi bee!, Aman Allah'ım, Aman Allahım, Allah Allah, Çok gürültülü Hava, Vay anasına, Vay canına, Muhteşem, Aman Tanrım, Hayret.

Wirt: Lokantacı, Mekansahibi, Ev sahibi, meyhaneci, Wirt, İşletmeci, Hancı, garson, bahçıvan.

Bewertung der Übersetzungen:

Pragmalinguistisch

In diesem Beispielsatz werden hauptsächlich die

Stilistische Bewertung Übersetzungserscheinungen der Begriffe Donnerwetter und Wirt bewertet. Das Idiom Donnerwetter, das in diesem Satz eine Aktion der Überraschung erklärt, ist ein pragmalinguistisches Element. Stilistisch sollte diese Aussage auch im Türkischen durch ein Idiom wiedergegeben werden. Aus den Übersetzungen der Bilingualen stellt sich fest, dass größtenteils darauf geachtet wird, die Idiome im Türkischen stilistisch äquivalent wiederzugeben (es zeigen sich häufig pragmatische Erscheinungen), doch werden auch Übersetzungen wie çok gürültülü hava (D. lärmendes Wetter) durchgeführt, die den pragmalinguistischen und stilistischen Eigenschaften des Wortes nicht äquivalent sind. Auch das Wort Wirt ist pragmalinguistisch aufgrund seiner Kulturbedingtheit schwer ins Türkische zu übertragen. Dieses Wort wird als Gastronom, Lokalbesitzer, Hausbesitzer, Unternehmer, Herbergsvater, Kellner oder Gärtner sehr unterschiedlich übersetzt oder in manchen Übersetzungen nicht beachtet und übersetzt. Bei beiden Begriffen handelt es sich um eine Eins-zu-Teilübersetzung. Pragmalinguistisch und semantisch betrachtet besteht daher keine eindeutige Äquivalenz. 


\subsection{Beispiel 3}

"Der Gong lärmte zur Abendmahlzeit." (Zweig, DM, s. 33) Übersetzungsbeispiele der Studierenden:

1. Akşam yemeğine zil sesi çınlıyordu.

2. Çan sesi akşam yemeği vaktinde çaldi.

3. Çan sesi çok gürültülüydü akşamyemeği zamanında.

4. Gong sesi Akşam yemeği saatin de çaldı.

5. Akşam yemeğinde çan gürültü yapıyordu.

6. Çan akşam yemeği saatini gürültüyle haber veriyordu.

7. Akşam yemeği esnasında zil sesi ortalığı inletti.

8. Çan akşam yemeği için ötüyordu.

9. Gong akşam yemeği için çalıyordu.

Bewertung der Übersetzungen:

\begin{tabular}{l|l} 
Pragmalinguistische & Der Gong bzw. der Glockenschlag ist ein deutsches kulturelles Element,
\end{tabular} Bewertung das im Türkischen keine sprachliche Entsprechung hat. Die durch die Eins-zu-Null-Entsprechung entstandene sprachliche Lücke wird von den meisten Studierenden mit einer Teilübersetzung wie çan sesi (D. Glockenton) oder zil sesi (D. Klingelton) geschlossen, oder der Begriff wird als Fremdwort in den Satz eingebaut bzw. ein intrasententiales Code-Switching durchgeführt. Als Zweites lassen sich Fehler in der Verwendung von Verben feststellen. Das Verb lärmen wird in der Zielsprache mit çınlamak, çalmak, gürültülü olmak, gürültü yapmak, haber vermek, inletmek, ötmek wiedergegeben. Die Verwendung dieser Verben zeigt, dass die Studierenden im Hinblick auf die pragmatischen Verwendungen meistens nicht äquivalente Begriffe wählen und im Übersetzungsprozess die konnotative Bedeutung beachtet haben.

Semantische Die Präposition zur wird von den Studierenden in verschiedenen

Bewertung Bedeutungen übersetzt (Bsp. esnasında; D. während), in dem die Aussage in der Zielsprache semantisch nicht äquivalent erscheint. Die Bestimmungen der Bilingualen weisen auf die fehlenden Grammatikkenntnisse in der Zielsprache hin.

\subsection{Beispiel 4}

">Gewiß gestatte ich, hehe ... alles gestatte ich ... was Sie wollen ... hehe ... alles gestatte ich ... alles, was ich habe, steht ja zu Ihrer Verfügung ... mit mir kann man sich alles gestatten ...<" (Zweig, UEH, s. 19)

Übersetzungsbeispiele der Studierenden:

1. Tabiki izin veriyorum, haha... herşeye izin veriyorum... ne isterseniz Нa Нa... herşeye, sahip olduklarım sizin hizmetinizde... Benimlen herşeyi yapabilirsiniz.

2. Tabii ki müsaade ederim, hahaha herşeye müsaade ederim... ne isterseniz... hahaha... herşeye müsaade ederim... herşeye, neyim warsa size tahsiz edilmiş durumda...benimle herşey yapılabilir.

3. "Tabiki izin veriyorum, $h_{1} h_{1} .$. herşeye izin veriyorum... ne isterseniz... $h_{1} \mathrm{~h}$ 1... herşeye izin veriyorum... herșeye, her neyim varsa, sizin emrinizdeyim... benimle herșey yapılabilir..."

4. Benimle her şeye izin verebilirsiniz.

5. Neyim var neyim yoksa emrinin altında..

6. Benden her șey için izin alabilirsiniz.... 


\begin{tabular}{|l|l|}
\hline \multicolumn{1}{|l|}{ Bewertung der Übersetzungen: } \\
\hline Bewertung & $\begin{array}{l}\text { In diesem Beispiel ist zu erwähnen, dass hinsichtlich der Orthographie } \\
\text { verschiedene Fehler festzustellen sind. Zu diesen Fehlern gehören sowohl } \\
\text { die Satzzeichen als auch die Rechtschreibfehler im Zieltext. Außerdem ist } \\
\text { zu erkennen, dass einige Studierende Buchstaben aus dem deutschen } \\
\text { Alphabet (wie im Bsp. 2- warsa) verwendet haben. Die falsche } \\
\text { Schreibweise des Wortes "Benimlen" (benimle - D. mit mir) zeigt } \\
\text { wiederum, dass eine falsche Aussprache vorhanden ist. Ein nächster } \\
\text { orthographischer Fehler ist die falsche Transkription des Wortes "tahsiz" } \\
\text { (tahsis - D. Zuweisung). Derartige Fehler weisen auch auf } \\
\text { Interferenzfehler und Probleme der Bilingualen bei der türkischen } \\
\text { Transkription. Zuletzt ist zu erwähnen, dass das Lachen wiedergebender } \\
\text { Ausruf hehe in den Übersetzungen mit verschiedenen Interjektionen und } \\
\text { manchmal nicht als Reduplikation wiedergegeben wird, obwohl im } \\
\text { Türkischen lautlich eine Eins-zu-Eins-Entsprechung dieser Interjektion } \\
\text { vorhanden ist. Deshalb sind die verschiedenen und nicht äquivalenten } \\
\text { Übersetzungen wie hr hı der Studierenden entweder willkürlich oder sie } \\
\text { beruhen auf fehlenden Sprachkenntnissen. }\end{array}$ \\
\hline
\end{tabular}

\subsection{Beispiel 5}

"Und Ihr wißt, auch diesmal hat die Mutter Gottes ein Wunder der Genesung dort gewirkt, wo alle Ärzte sich mit Verzweiflung abgewandt hatten." (Zweig, DWDL, s. 14-15)

Übersetzungsbeispiele der Studierenden:

1. Ve sizde biliyorsunuz ki, bütün doktorların umutsuz bir şekilde döndüğü yerde, Meryem ana orada bir mucize gerçekleştirdi.

2. Biliyorsunuz, bu seferde bütün hekimlerin çaresizlikten yüz çevirdiği şifayı tanrı annesinin mucizesi nekahete etki etti.

3. Ve biliyorsunuz, doktorlar umutlarını kestikleri halde, bu seferkinde de Anne, Tanrı'nın bir mucizesi sayesinde orda şıfa buldu.

4. Ve biliyorsunuz, bu sefer de Tanrı bir mucize sundu ve doktorların umutsuzca yüz çevirdikleri zaman, iyileştirdi.

5. Ve siz biliyorsunuz, bu sefer de Tanrı ana bir mucize ile iyileşmeleri orda işe yaradı, tüm doktorlar ümitsizce sırtını çevirirken.

6. Ve bu sefer de biliyorsunuz, Tanrı'nın annesi orada bir iyileşme mucizesi yarattı, tüm doktorların umutsuzlukla baktı̆̆ 1 yerde.

7. Ve bildiğiniz gibi Meryemana bu kez orada bir şifalanma mucizesi yarattı ki tüm doktorlar çaresizlik içinde yüzlerini çevirmişti.

8. Ve bildiğiniz üzere, bu sefer de Allah'ın mucizesiyle annesi iyileşti, tam da bütün doktorların çaresizlik içinde pes ettikleri esnada.

Bewertung der Übersetzungen:

\begin{tabular}{|l|l|l}
\hline Pragmalinguistische & In diesem Beispiel wird insbesondere auf die pragmatische und
\end{tabular} Bewertung semantische Äquivalenz geachtet. Das kulturbedingte Wort Mutter Gottes wird von den Studierenden als Meryem Ana (D. Mutter Maria), Tanrı ana (D. Mutter Gott), Tanrı (D. Gott), Tanrı'nın annesi (D. Gottes Mutter) ect. übersetzt. Damit wird gewiss Mutter Jesus d.h. Maria gemeint. Die richtige Übersetzung wäre dann Meryem Ana. Daher sind 


\begin{tabular}{|c|c|}
\hline & $\begin{array}{l}\text { die letzten drei Übersetzungen pragmalinguistisch falsch, da im } \\
\text { Türkischen für Mutter Gottes das Bezeichnung Meryem Ana gebraucht } \\
\text { wird. Bei einer Übersetzung muss man auch darauf achten, wo und wie } \\
\text { ein Wort pragmalinguistisch gebraucht wird. Soweit aus den } \\
\text { Übersetzungen zu erkennen, sind die Studierenden sprachlich nicht in } \\
\text { der Lage, dieses Wort im Zieltext richtig zu gebrauchen. }\end{array}$ \\
\hline $\begin{array}{l}\text { Semantische } \\
\text { Bewertung }\end{array}$ & $\begin{array}{l}\text { In den letzten zwei Beispielen wird der Satz von den Studierenden } \\
\text { semantisch betrachtet und übersetzt. Nach einer Rückübersetzung lässt } \\
\text { sich das sogleich feststellen. Bei der achten Übersetzung heißt es z.B.: bu } \\
\text { sefer de Allah'ın mucizesiyle annesi iyileşti. (Rückübersetzung: Auch diesmal } \\
\text { ist seine Mutter durch die Wunder Gottes genesen). Dass dieser Satz den } \\
\text { Studierenden schwer zu verstehen und wiederzugeben ist, ist von den } \\
\text { falschen Übersetzungen von Verben wie dönmek (D. sich wenden), yüz } \\
\text { çevirmek (D. sich abkehren), sırtını çevirmek (D. den Rücken drehen), } \\
\text { umutsuzlukla bakmak (D. hoffnungslos zusehen), pes etmek (D. aufgeben) } \\
\text { zu erkennen. Diese Fehler weisen auf die typischen Sprachfehler der } \\
\text { bilingualen Studierenden in der Zielsprache. }\end{array}$ \\
\hline Syntaktische Bewertung & $\begin{array}{l}\text { Der Satz, der aus einem Hauptsatz und einem Nebensatz aufgebaut ist, } \\
\text { ist syntaktisch ins Türkische äquivalent zu übertragen oder der } \\
\text { Nebensatz kann vor dem Verb eingebaut werden, ohne einen } \\
\text { satzsemantischen Fehler zu verursachen. Beim fünften Beispiel hat man } \\
\text { einen anderen d.h. temporalen Nebensatz gebildet (tüm doktorlar } \\
\text { ümitsizce sırtını çevirirken) und daher eine andere Konjunktion also } \\
\text { während gebraucht. Beim dritten Beispiel wird mit der Konjunktion } \\
\text { obwohl (doktorlar umutlarıı kestikleri halde) übersetzt. Es wird die } \\
\text { Reihenfolge der Haupt- und Nebensätze geändert oder sogar } \\
\text { Relativsätze (bütün hekimlerin çaresizlikten yüz çevirdiği şifayı) werden } \\
\text { gebildet. Das alles ist auch ein Zeichen dafür, dass die bilingualen } \\
\text { Studierenden nicht über genügende Kompetenz im syntaktischen } \\
\text { Bereich der Zielsprache verfügen. }\end{array}$ \\
\hline
\end{tabular}

\subsection{Beispiel 6}

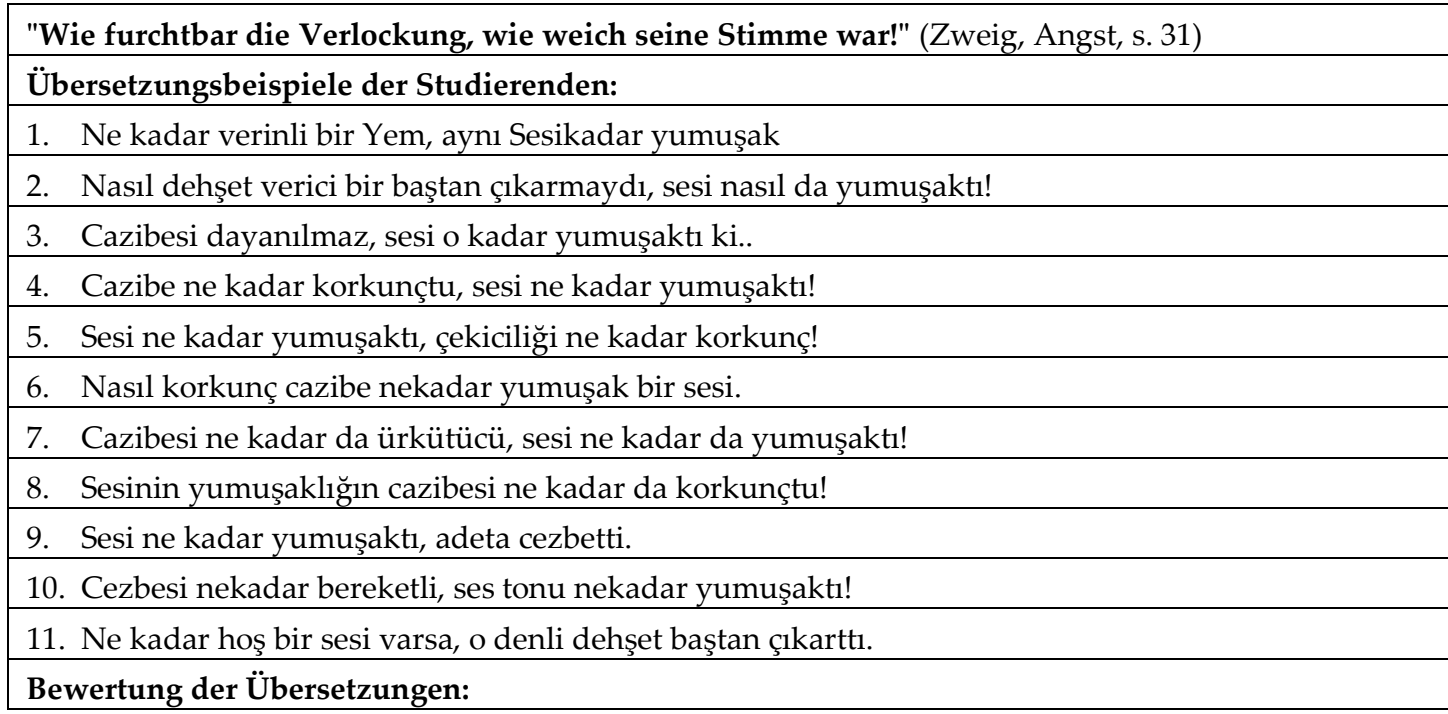




\begin{tabular}{|c|c|}
\hline Stilistische Bewertung & $\begin{array}{l}\text { Bei den Übersetzungen dieses Ausrufesatzes wird von den Studierenden } \\
\text { erwartet, dass sie auf die Stilistik dieser Aussage achten und die } \\
\text { Wortwiederholung am Anfang des Satzes im Türkischen äquivalent } \\
\text { auftritt. Die mit wie aufgebaute Anapher wurde jedoch von den } \\
\text { Studierenden meistens nicht beachtet. Somit ist zu betonen, dass sich in } \\
\text { den Übersetzungen die Struktur und der Rhythmus geändert haben. Der } \\
\text { Satz könnte stilistisch wie folgt übersetzt werden: "Ne kadar korkunç bir } \\
\text { baştan çıkarmaydı, ne kadar yumuşaktı sesi!" Hier handelt sich also um zwei } \\
\text { Ausrufesätze, daher müsste man sie im Türkischen mit zwei } \\
\text { Ausrufesätzen wiedergeben. Beim neunten Beispiel wird aber der zweite } \\
\text { Ausrufesatz wie ein normaler Aussagesatz (Sesi ne kadar yumuşaktı, adeta } \\
\text { cezbetti.) wiedergegeben. Aus diesem Grund ist diese Übersetzung } \\
\text { sowohl semantisch und syntaktisch als auch pragmalinguistisch und } \\
\text { stilistisch nicht äquivalent. Eine richtige Übersetzung dieses Satzes } \\
\text { verlangt in diesem Sinne Kenntnisse über die Syntaxregeln der } \\
\text { Zielsprache. }\end{array}$ \\
\hline $\begin{array}{l}\text { Semantische } \\
\text { Bewertung }\end{array}$ & $\begin{array}{l}\text { Das Adjektiv furchtbar ist aus semantischer Perspektive ein Problem für } \\
\text { die Studierenden bei dem Übersetzungsprozess. Das Adjektiv wird vom } \\
\text { Autor verwendet, um den Grad und die Stärke der Verlockung zu } \\
\text { betonen. Die Studierenden wählen dafür die Adjektive wie verimli ( } D \text {. } \\
\text { ergiebig, fruchtbar), dayanılmaz ( } D \text {. unwiderstehlich), ürkütücü (D. } \\
\text { unheimlich, beängstigend), bereketli (D. segensreich) und dehşet (D. } \\
\text { fürchterlich, grausig). Und daran kann man ohne weiteres erkennen, dass } \\
\text { Sprachkompetenz der Studierenden in semantischer Hinsicht nicht } \\
\text { ausreichend ist, wodurch selbstverständlich semantische Fehler } \\
\text { verursacht werden. }\end{array}$ \\
\hline $\begin{array}{l}\text { Orthographische } \\
\text { Bewertung }\end{array}$ & $\begin{array}{l}\text { Orthographisch betrachtet kommen auch hier viele Übersetzungsfehler. } \\
\text { Da es sich um zwei Ausrufesätze handelt, muss der Satz mit } \\
\text { Ausrufezeichen versehen werden. Wenn man jedoch die Sätze näher } \\
\text { betrachtet, ist festzustellen, dass die Satzzeichen nicht in allen } \\
\text { Übersetzungen äquivalent verwendet werden. Wie aus dem 1., 3., 6., 9. } \\
\text { und 11. Beispiel entnehmen ist. Abgesehen davon sind } \\
\text { Rechtschreibfehler wie Groß- und Kleinschreibung oder Zusammen- und } \\
\text { Getrenntschreibung vorhanden. Dafür einige Beispiele: verinli (T. } \\
\text { verimli), Yem (T. yem), Sesikadar (T. sesi kadar), nekadar (T. ne kadar), } \\
\text { Cezbesi (T. cazibesi). Diese Fehler weisen darauf hin, dass die bilingualen } \\
\text { Studierenden die orthographischen Regeln des Türkischen nicht } \\
\text { beachten oder Wissen über diese verfügen. }\end{array}$ \\
\hline
\end{tabular}




\subsection{Beispiel 7}

"> Na ... zum Beispiel der Ring da... [...] ich hab' ja nie einen gehabt...[...]<" (Zweig, Angst, s. 54) Übersetzungsbeispiele der Studierenden:

1. >Aynı... burdakiörnek Yüzük gibi...[...] benim hiç olmamıştı<

2. "Şöyle...Hiçbir zaman sahip olmadığım ... örneğin oradaki yüzük gibi"

3. "Mesela... Parmağınızdaki yüzlük. Hiç öyle bir yüzüğüm olmamıştı."

4. Örneğin surdakı yüzükten benim hiç olmadı.

5. Örneğin ordaki yüzük... benim hiç olmadı ya...

6. "Em... bu yüzük mesela... [...] hiçbir zaman birine sahip değildim..."

Bewertung der Übersetzungen:

Stilistische Bewertung

Dieser elliptische Satz ist ein Beispiel der Alltagssprache, deshalb wird von den Studierenden erwartet, dass sie diese Aussagen in der Zielsprache als elliptische Sätze wiedergegeben und die Übersetzung insbesondere syntaktisch und stilistisch dem Original treu bleibt. Bei der Analyse der Übersetzungen stellt sich jedoch fest, dass die Sätze im ersten Beispiel als elliptischer Satz und im zweiten Beispiel als Relativsatz (Hiçbir zaman sahip olmadığım ... örneğin oradaki yüzük gibi) wiedergegeben werden, wodurch das Wort Ring erklärt wird. Infolgedessen werden die Sätze syntaktisch und stilistisch nicht äquivalent in die Zielsprache übertragen.

Semantische In diesem Beispiel beginnt der Satz mit einer Interjektion, die ins

Bewertung Türkische zu übersetzen ist, destotrotz wird dieser Element in einigen Übersetzungen weggelassen, weshalb eine Lücke entsteht. Die Übersetzungen Aym (D. Gleich), Şöyle (D. So), Mesela (D. zum Beispiel), Örneğin (D. zum Beispiel) entsprechen semantisch nicht der Aussage. Die Übersetzung $E m$ im sechsten Beispiel entspricht genauso nicht der Bedeutung der deutschen Interjektion und ist ein Beispiel zum Interferenzfehler, da sie im türkischen Sprachgebrauch nicht existiert. Im zweiten Satzteil wird für den Ring ein unbestimmter Artikel verwendet, der im Türkischen schwer $\mathrm{zu}$ übersetzen ist. In den meisten Übersetzungen wird deshalb das Wort yüzük (D. Ring) hinzugefügt. Es ist zu markieren, dass diese Anfügung die Satzsemantik bekräftigt.

\subsection{Beispiel 8}

">Geben S'mir doch auch die Silbertasche, damit ich das Geld nicht verlier'!<" (Zweig, Angst, s. 18) Übersetzungsbeispiele der Studierenden:

1. Bana da gümüş çantayı versenize, parayı kaybetmemem için!

2. Gümüş çantasını da verin ki Parayı kayıp etmiyim.

3. "Gümüş çantayı da verirmisiniz, parayı kaybetmeyeyim"

4. Parayı kaybetmemem için bana gümüş çantayı da verir misiniz!

5. "Bana gümüş çantayıda verin, parayı kaybetmemek için!"

Bewertung der Übersetzungen:

Stilistisch-

Syntaktische

Bewertung
In diesem Beispiel handelt es sich um einen Imperativsatz, der mit einem Nebensatz endet. Aus orthographischer Betrachtung endet der erste, vierte und fünfte Satz mit dem richtigen Satzzeichen, einige Sätze 


\begin{tabular}{|l|l|}
\hline dagegen mit einem Punkt. Außerdem wird im dritten Beispiel der erste \\
(Gümüş çantayı da verirmisiniz) und im vierten Beispiel der zweite Satzteil \\
(bana gümüş̧̧̧ntayı da verir misiniz!) als Fragesatz gebildet. Die zweite \\
Übersetzung zeigt einen syntaktischen Fehler, indem der Satz nicht mit \\
damit, sondern so dass wiedergegeben wird. Ein weiterer syntaktischer \\
Fehler ist im Beispiel drei vorhanden, in dem die Übersetzung mit zwei \\
Hauptsätzen wiedergegeben wird, was syntaktisch, semantisch und \\
stilistisch falsch ist. Außerdem wird die Partikel doch in den meisten \\
Übersetzungen nicht beachtet. Die stilistische Aussage mit Beispielen von \\
Apokope ist aufgrund der Vokalregeln im Türkischen schwer mit \\
denselben rhetorischen Figuren wiederzugeben. Doch satzsemantisch \\
und syntaktisch ist dieser Satz äquivalent zu übersetzen.
\end{tabular}

\subsection{Beispiel 9}

"[...] wann sie nur in ihrem blaßblauen Kleidchen von der Schule zum Zuckerbäcker geführt, den Kinderkuß gefühlt von dem noch bezuckerten Mund [...]" (Zweig, UEH, s. 8)

Übersetzungsbeispiele der Studierenden:

1. blaßblauen: soluk mavi, bebek mavisi, rengi kaçmıs mavi, açık mavi, masmavi

2. Kleidchen: elbisesi, kiyafet

3. Zuckerbäcker: şekerlemeci, fırın, pastane

4. Kinderkuß: çocuksu bir öpücük/buse, çocuksu öpücük, Kinderkuß, çocuk öpücüğü, çocukça masum buse

\section{Bewertung der Übersetzungen:}

Semantische

Bewertung
Der Begriff blassblau wird von den meisten Studierenden mit soluk mavi semantisch korrekt übersetzt. Auch zeigt sich, dass die Studierenden die Transkription nach den türkischen Sprachregeln führen und die beiden Adjektive auseinander schreiben. Dennoch gibt es auch Übersetzungsbeispiele wie bebek mavisi (D. Babyblau), rengi kaçmış mavi (D. verfärbtes Blau), açık mavi (D. Hellblau), masmavi (D. blau konsolidierend). Das Zusammengesetzte Wort Zuckerbäcker besitzt im Türkischen eine Eins-Zu-Teil-Entsprechung Şekerlemeci, deren Bedeutung Konditor ist. Dieses heutzutage weniger gebrauchte Wort wird von einer Mehrzahl als firın (D. Bäckerei) oder pastane (D. Bäckerei) in die Zielsprache übertragen. Im letzten Beispiel werden verschiedene Übersetzungen geführt, die semantisch dem zusammengesetztem Wort Kinderkuss entsprechen. In einer Übersetzung erscheint ein intrasententiales Code-Switching bzw. wird das deutsche Wort in die türkische Übersetzung eingebaut. Außerdem gibt es auch Beispiele, in denen das Ziel ist, die Semantik des zweiten Satzteiles mit Adjektiven wie masum (D. harmlos) zu verstärken.

Morphologische Bei der Übersetzung des Wortes Kleidchen wird von mehreren Studierenden nicht auf die Diminutivendung geachtet, obwohl die Suffixe -cık/-cik semantisch dieser Form entsprechend sind. Als Zweites gibt es die Möglichkeit, diese Verkleinerungsform mit dem Adjektiv minik (D. winzig) wiederzugeben. 


\subsection{Beispiel 10}

">Entschuldigen scho, gnä’ Herr<, sagte sie, mit dem Blick auf dem Boden herumwischend." (Zweig, DM, s. 66)

Übersetzungsbeispiele der Studierenden:

1. "affedersiniz sayın bayım", diyordu yere süzüle süzüle bakarak.

2. >Özür Dilerim Bayım< diyordu, yere bakan temizleyici bakışları ile.

3. "özürdilerim sayın bay" bakışlarını yere kaydırarak dedi.

4. Zoraki bir şekilde, bakışlarıyla yeri süzerek, >özür dilerim bayım< diye mırıldandı.

5. >Özrümü dilerim beyim< diyordu, yerde bi o yana bir diğer yana bakan bakışları ile.

6. "Affedin, merhametli Beyefendi", yeri süzerek söyledi kız.

7. "Kralarlar tab, say' bayım", dedi yerlerde bakışlarını gezdirirken.

8. Affedersiniz beyefendi. Bakışı yerlerde sürünürken söyledi.

Bewertung der Übersetzungen:

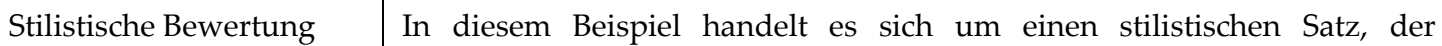
alltagssprachlich aufgebaut ist. Dadurch liegt der Schwerpunkt bei der Analyse der Übersetzungen hauptsächlich auf den stilistischen Elementen. Bei den meisten Übersetzungen stellt sich fest, dass die Studierenden Probleme bei den Wiedergaben dieser stilistischen Eigenschaften haben. Die hochsprachige Anredeform ist im Türkischen mit Saygıdeğer wiederzugeben. Die Studierenden wählen für die Wiedergabe die türkischen Begriffe sayın (D. sehr geehrter), merhametli (D. barmherziger) oder übersetzen dieses Wort nicht, wodurch eine semantische Lücke entsteht.

Semantische Bewertung Aus semantischer Perspektive ist die Art der Wiedergabe des Adjektivs herumwischend von enormer Bedeutung. Die Übersetzungen sind wie folgt: süzüle süzüle ( $D$. Diese Reduplikation könnte als langsam verstanden werden), temizleyici (D. reinigend), kaydırarak (D. verschiebend), bir o yana bir diğer yana bakan (D. Mal auf diese oder jene Seite schauenden), gezdirirken (D. umherblickenden), sürünürken (D. kriechenden). Nach einer Bewertung stellt sich fest, dass die Studierenden dieses Element semantisch nicht äquivalent wiedergeben können. Eine äquivalente Übersetzung des Satzteils "mit dem Blick auf dem Boden herumwischend" wäre in der Zielsprache bakışları yeri süzerek. 


\section{CONCLUSION}

Die Übersetzung eines Ausgangstextes in eine Zielsprache beeinflussen mehrere Faktoren wie die Gattung und die Länge des zu übersetzenden Textes, die Zeitspanne, Hilfsmaterialen oder Quellen etc. Jedoch ist zu erwähnen, dass die Übersetzung von literarischen Texten hauptsächlich mehrere Eigenschaften vom Übersetzer verlangen. Dazu gehören vor allem Kenntnisse, zu denen Sprachwissen, Normwissen, Kompetenz und Interesse gehören.

Diese Arbeit hat es zum Ziel den sprachlichen Makeln der bilingualen Studierenden zu bestimmen. Dazu wurden aus drei verschiedenen Universitäten [Ankara Universität (Ankara), Hacettepe Universität (Ankara), Akdeniz Universität (Antalya)] bilinguale Studierende ausgewählt, von denen erwartet wurde, dass sie Sätze aus verschiedenen Werken von Stefan Zweig übersetzen. Da der Fachbereich der Studierenden Germanistik ist, sind ihnen die Werke und die stilistischen Eigenschaften Zweigs bekannt. Außerdem haben alle Studierende mindestens einen Übersetzungskurs besucht. Die Untersuchungsgruppe besteht somit aus kompetenten Bilingualen, die die deutsche Sprache ungesteuert erlernt haben und in einer türkischen Universität Germanistik studieren. Die Frage war, wie die bilingualen Studierenden die kommunikativen Möglichkeiten in einer literarischen Übersetzung gebrauchen werden und welche Fehler in Erscheinung kommen würden. Aus zehn Satzübersetzungen ohne Hilfsmaterial stellte sich fest, dass Interferenzfehler häufig vorkommen. Diese sind schwerwiegend orthographische Fehler wie der falsche Gebrauch von Satzzeichen, die Regeln der Groß- und Kleinschreibung oder Zusammen- und Getrenntschreibung und sowie die falsche Transkription in der Zielsprache. In den Übersetzungen waren häufig willkürliche Wortwahlen zu erkennen, obwohl es bei meisten Wörtern semantisch äquivalente Begriffe in der Zielsprache gibt. Diese Wahl der Studierende führt $\mathrm{zu}$ fehlerhaften semantischen Wiedergaben. Insbesondere bei pragmatischen Äußerungen kommt es öfters vor, dass diese direkt übersetzt wird, wobei eine semantische Lücke entsteht. Syntaktisch versuchen die Studierenden eine äquivalente Reihung zu erschaffen, in diesem Sinne ist zu erwähnen, dass dies den mehreren gelungen ist. Die sprachlichen Regeln der Zielsprache apropos den orthographischen Eigenschaften wurden im großen Maße beachtet. Die meisten Studierenden achten auch auf die Sprachart des Ausgangstextes und übersetzen die Textteile stilhaft. Bei Redewendungen jedoch zeigt sich deutlich, dass die Bilingualen aufgrund von wenigem Gebrauch derselben, nicht immer fähig sind, diese in der richtigen Bedeutung und als Redewendung wiederzugeben. Zuletzt ist zu erwähnen, dass sich das Niveau der Fähigkeit, Fertigkeit und Kompetenz der Studierenden sich entsprechen, doch der Übersetzungspotenzial sich abweicht. Der Gebrauch des Code-Switching kommt auch in mehreren Übersetzungsbeispielen in Erscheinung. Dieser ist ein Zeichen, dass die Bilingualen bei einer Lücke im Wortschatz auf den Begriff im Ausgangstext angreifen, ohne das Wort zu erklären oder Informationen hinzuzufügen. Im Generellen ist auch zu erkennen, dass sich Schwierigkeiten bei der Satzsemantik zeigen. In diesem Fall wird entweder der Ausgangstextteil nicht verstanden oder der Textinhalt aus Mangel an sprachlichen Wissen nicht in der Zielsprache wiedergegeben. Die Fehler, die im Allgemeinen auftreten, sind vor allem orthographisch, semantisch, syntaktisch, stilistisch und pragmatischer Art, die sich allerdings wegen der Zweisprachigkeit zum Teil auf die Interferenzfehler beziehen, weil sie die ausgangssprachlichen Regeln in die Zielsprache übertragen, wobei die Übersetzung negativ beeinflusst wird und viele Übersetzungsfehler vorkommen. 


\section{SUMMARY}

Bilingual people are able to understand content in two languages and to communicate in both languages. In a translation process, however, it is not enough to master two languages, as the translation of a source text into the target language requires additional skills in addition to language knowledge. Especially in translations of literary texts, in which the style and genre can be very important, errors often occur with bilingual people. In addition to the stylistic features that must be observed, other linguistic norms are also important. A literary text cannot and should not be roughly translated, but appear as faithfully as possible, as if reading the text in the original language is like an illusion. Here it is of course important to use the right words that are semantically equivalent. It is also important that the syntax - if possible - be set up immediately and e.g. reproduces a rhetorical sentence in the same rhetorical way. The aim of this thesis is to analyze errors based on literary translations and to identify the types of errors frequently made by bilingual people. For this purpose, students from three different universities are selected and expected to translate extracts from Stefan Zweig's various works. The students translated a total of ten sentences, which are evaluated from a stylistic, orthographic, semantic, syntactic, morphological and pragmatic perspective. On the subject of this procedure, attention is also paid to Werner Koller's correspondence types. From ten sentence translations without auxiliary material it was found that interference errors occur frequently. These are serious spelling mistakes such as the incorrect use of punctuation marks, the rules of upper and lower case or compound and hyphenation, and incorrect transcription in the target language. In the translations, arbitrary word choices have often been seen although most words have semantically equivalent terms in the target language. Most students also pay attention to the language of the source text and translate the text parts stylishly. Finally, it should be mentioned that the level of ability, skill and competence of the students correspond, but the translation potential differs. The use of code switching also appears in several translation examples.

Article Information

\begin{tabular}{ll}
\hline Ethics Committee Approval: & Exempt from the Ethics Committee Decision. \\
Informed Consent: & No participant \\
Financial Support: & No financial support from any institution or project. \\
Conflict of Interest: & No conflict of interest. \\
Copyrights: & No material subject to copyright is included.
\end{tabular}

SEFAD, 2021; (45): 63-80 


\section{BIBLIOGRAPHY}

Banaz, H. (2002). Bilingualismus und Code-switching bei der zweiten türkischen Generation in der Bundesrepublik Deutschland. Sprachverhalten und Identitätsentwicklung. Essen: Linguistik Server.

Benteler, A. (2019). Sprache im Exil. Mehrsprachigkeit und Übersetzung als literarische Verfahren bei Hilde Domin, Mascha Kaléko und Werner Lansburgh. Berlin: J.B. Metzler.

Best, O.F. (2004). Handbuch Literarischer Fachbegriffe. Frankfurt am Main: Fischer Taschenbuch.

Greiner, N. (2004). Übersetzung und Literaturwissenschaft. Tübingen: Gunter Narr Verlag.

Hacısalihoğlu, E. (2009). >Türkisch-deutsche Sprachalternationen im Grundschulalter.<. Neumann, U. \& Reich H.H. (Ed.), Erwerb des Türkischen in einsprachigen und mehrsprachigen Situationen içinde (91-128). Münster: Waxmann Verlag.

Hansen, G. (1999). >Die Rolle der fremdsprachlichen Kompetenz.<. Snell-Hornby, Mary \& Hönig, H. G. \& Kußmaul, P. \& Schmitt, P.A. (Ed.), Handbuch Translation içinde (341343). Tübingen: Stauffenburg Verlag Brigitte Narr GmbH.

Klein, W. (1992). Zweitsprachenerwerb. Frankfurt a.M.: Verlag Anton Hain.

Koller, W.(2001). Einführung in die Übersetzungswissenschaft. Wiebelsheim: Quelle\&Meyer Verlag.

Lewandowski, T. (1979). Linguistische Wörterbuch 1. Heidelberg: Quelle \&Meyer Verlag.

Löffler, H. (2010). Germanistische Soziolinguistik. Berlin: Erich Schmitt Verlag.

Ortega, L.(2009). Understanding Second Language Acquisition. New York: Routledge.

Reinart, S. (2009). Kulturspezifik in der Fachübersetzung. Berlin: Frank\&Timme GmbH Verlag.

Resch, R. (1999). >Die Rolle der muttersprachlichen Kompetenz.<. Snell-Hornby, Mary \& Hönig, H.G. \& Kußmaul, P. \& Schmitt, P.A. (Ed.), Handbuch Translation. içinde (343345). Tübingen: Stauffenburg Verlag Brigitte Narr GmbH.

Stolze, R. (2008). Übersetzungstheorien. Eine Einführung. Tübingen: Gunter Narr Verlag.

Stolze, R. (2013). Fachübersetzen - Ein Lehrbuch für Theorie und Praxis. Berlin: Frank\&Timme GmbH Verlag.

Zweig, S. (t.y.). Angst. İstanbul: Karbon Kitaplar.

Zweig, S. (t.y.). Die Mondscheingasse. İstanbul: Karbon Kitaplar.

Zweig, S. (t.y.). Die Wunder des Lebens. İstanbul: Karbon Kitaplar.

Zweig, S. (t.y.). Untergang eines Herzens. İstanbul: Karbon Kitaplar. 